Classification

Physics Abstracts

81.10.Aj-61.50.Ks

\title{
HRTEM Study of AlN Formed by Nitrogen Implantation in Al Films
}

\author{
Zineb Seghrouchni, Lina El Chahal, Aimé Mosser and Gabrielle Ehret \\ IPCMS-GSI, UMR 046 du CNRS, 23 rue du Loess, 67037 Strasbourg Cedex, France
}

(Received May 15; accepted September 24, 1996)

\begin{abstract}
Résumé. - On a analysé par microscopie électronique à haute résolution des films d'aluminium de $70 \mathrm{~nm}$ d'épaisseur déposés sur des grilles en cuivre et implantés à $20 \mathrm{keV}$ avec des doses égales à $1 \times 10^{17}, 2 \times 10^{17}$ et $3 \times 10^{17} \mathrm{~N}^{+} / \mathrm{cm}^{2}$. A faible dose, la phase cubique est prépondérante et se présente sous forme de grains de taille importante et de morphologie mal définie. Au voisinage de ces grains, de petits cristallites (embryons) de AlN de structure hcp sont précipités. Lorsqu'on augmente la dose jusqu'à $3 \times 10^{17} \mathrm{~N}^{+} / \mathrm{cm}^{2}$, les petits cristallites de AlN hcp croissent pour devenir des cristallites facettés ayant une taille de 15 à $30 \mathrm{~nm}$ et des orientations préférentielles [0001], [1102] et [1100]. En même temps, la structure cubique de certains grains de morphologie mal définie se transforme en structure hcp. Par ailleurs, l'étude EDS effectuée sur les sections planes et transversales, a permis de localiser l'azote et de quantifier les rapports de concentration N/Al.
\end{abstract}

\begin{abstract}
Aluminum thin films ( $70 \mathrm{~nm}$ thick) deposited on a copper grid and then nitrogen implanted at $20 \mathrm{keV}$ with doses of $1 \times 10^{17}, 2 \times 10^{17}$ and $3 \times 10^{17} \mathrm{~N}^{+} / \mathrm{cm}^{2}$ are analyzed by HRTEM techniques. At low dose $\left(1 \times 10^{17} \mathrm{~N}^{+} / \mathrm{cm}^{2}\right)$, it is shown that the cubic phase is preponderant, while small AlN particles with hcp wurtzite-type (1010) planes are present in the vicinity of grains of more important size with cubic structure and ill-defined morphology. When increasing the dose up to $3 \times 10^{17} \mathrm{~N}^{+} / \mathrm{cm}^{2}$, AlN particles grow into faceted crystallites (15-30 nm size) with [0001], [i1102] and [1100] preferred orientation, while the fcc structure of some ill-defined grains transforms to hcp. Besides, an EDS study, on both parallel and cross sections, was realized in order to localize nitrogen and to quantify N/Al ratios.
\end{abstract}

\section{Introduction}

Aluminum nitride thin films are used in a number of technological applications (passivation of semiconductors, transmission of surface acoustic waves, ...), where their hardness, their anticorrosive and electrical insulating properties and their stability at high temperature are useful.

AlN production has been realized by several processes (chemical vapor deposition [1], reactive sputtering [2], reactive ion plating [3] ...) which involve usually high temperatures during the growth and need subsequent annealing in order to well crystallize the films. Besides, there 
have been several reports [4-7] in the literature on the direct formation of AlN by nitrogen ion implantation in $\mathrm{Al}$ metal.

These results are partly contradictory, since Pavlov et al. [4], Rauschenbach et al. [6] and Ohira and Iwaki [7] have shown by electron and X-ray diffraction the formation of hexagonal AlN in deposited thin films or aluminum matrices by ion implantation at doses ranging from $1 \times 10^{16}$ to $1 \times 10^{18} \mathrm{~N}^{+} / \mathrm{cm}^{2}$ and at implantation energies of 50 to $150 \mathrm{keV}$, while Bykov et al. [8] were unable to detect this nitride after implantation at doses ranging from $1 \times 10^{15}$ to $1 \times 10^{18} \mathrm{~N}^{+} / \mathrm{cm}^{2}$ and at energies going from 30 to $50 \mathrm{keV}$.

Furthermore, contrary to the majority of the preceding reports, Matthews et al. [9] have shown the formation of both fcc (lattice parameter $=7.91 \AA$ ) and hexagonal nitrides by nitrogen implantation with doses ranging from $10^{17}$ to $10^{18} \mathrm{~N}^{+} / \mathrm{cm}^{2}$ and using energies comprised between 25 and $75 \mathrm{keV}$. It should be noticed that two different AlN compounds of fcc structure are given in the ASTM tables $\left({ }^{1}\right)$, one having $4.12 \AA$ and the other $7.91 \AA$ as lattice parameter. Furthermore, these compounds have not been studied extensively and especially were not reported in [4-8].

In order to try clarifying the aluminum nitride formation conditions with regard to the previous works, we report, in this paper, a detailed high resolution transmission electron microscopy (HRTEM) study of nitrogen implanted in aluminum thin layers with the help of EDS microanalysis. Especially we were interested by the microstructural state of implanted $\mathrm{Al}$ in the earlier stage of the hcp AlN grain growth. In a previous paper [10], we realized a X-ray diffraction study of $60 \mathrm{keV}$ nitrogen implanted $\mathrm{Al}$ layers deposited on glass plates. We pointed out that a cubic solid solution Al-N was formed for all implantation doses $\left(1,3,5 \times 10^{17} \mathrm{~N}^{+} / \mathrm{cm}^{2}\right)$ and that a hcp AlN compound was formed at the highest dose.

\section{Experimental Details}

Aluminum films, $70 \mathrm{~nm}$ thick, were evaporated onto copper grids pre-coated with a supporting carbon film $(10 \mathrm{~nm})$. During deposition, the residual vacuum was better than $2 \times 10^{-6}$ mbar.

Nitrogen ion implantation at $20 \mathrm{keV}$ was performed with an EATON $200 \mathrm{MC}$ ion implanting machine with a beam current density of $4 \mu \mathrm{A} / \mathrm{cm}^{2}$. Samples were implanted over the dose range 1 to $3 \times 10^{17} \mathrm{~N}^{+} / \mathrm{cm}^{2}$ with a pressure better than $1 \times 10^{-6}$ mbar in the target chamber. The projected range and straggle of the $\mathrm{N}^{+}$ions, calculated using a TRIM program [11], are respectively $50 \mathrm{~nm}$ and $20 \mathrm{~nm}$. The calculated maxima concentrations ratios $\mathrm{N} / \mathrm{Al}$ for $1 \times 10^{17}, 2 \times 10^{17}$ and $3 \times$ $10^{17} \mathrm{~N}^{+} / \mathrm{cm}^{2}$ are respectively $0.32,0.65$ and 0.97 . The HRTEM observations were made with a TOPCON 002B ultra high resolution microscope operating at $200 \mathrm{kV}$. The point-to-point limit resolution is $0.18 \mathrm{~nm}$ and the simulation of the experimental micrographs, based on the multislice method, is performed with an EMS software [12]. Selected area (400 nm) and microbeam (4.2$31 \mathrm{~nm}$ ) diffraction patterns combined with EDS analysis extended to light elements were obtained. In order to improve the intensity yield of light elements for EDS analysis, the samples were tilted by $10^{\circ}$ and the acquisition time was extended to $300 \mathrm{~s}$.

Cross section cuts were realized through the $\mathrm{Al}$ implanted samples, previously incorporated in polymerized epoxy EPON resin, using an ultramicrotome.

$\left({ }^{1}\right)[25-1495]$ fcc AlN, $a_{0}=4.12 \AA$. [34-679] fcc AlN, $a_{0}=7.91 \AA$. 
Table I. - Lattice: Parameter of aluminum and fcc aluminum nitride.

\begin{tabular}{|l|c|c|c|}
\hline & fcc Al & fcc AlN & fcc AlN \\
\hline$a_{0}(\AA)$ & 4.049 & 4.12 & 7.91 \\
\hline
\end{tabular}

Table II. - Interplanar distances of Al and AlN.

\begin{tabular}{|c|c|c|c|c|c|}
\hline $\begin{array}{c}\text { dose } \\
\left(\mathrm{N}^{+} / \mathrm{cm}^{2}\right)\end{array}$ & $\begin{array}{c}d^{\exp }(h k l) \\
(\mathrm{nm})\end{array}$ & fcc Al & fcc AlN ** & hcp AlN *** & $h k l(i)$ \\
\hline $3 \times 10^{17}$ & 0.267 & & & 0.269 & 1000 \\
\hline & & & & 0.249 & 0002 \\
\hline $1 \times 10^{17}$ & 0.237 & 0.236 & 0.238 & & $\begin{array}{c}10 \overline{1} 1 \\
111\end{array}$ \\
\hline $1 \times 10^{17}$ & 0.207 & 0.202 & 0.206 & & 200 \\
\hline $2 \times 10^{17}$ & 0.180 & & & 0.183 & $10 \overline{1} 2$ \\
\hline $2 \times 10^{17}$ & 0.154 & & & 0.155 & $11 \overline{2} 0$ \\
\hline $2 \times 10^{17}$ & 0.143 & & & 0.148 & $11 \overline{2} 1$ \\
& & 0.143 & 0.146 & & 220 \\
\hline
\end{tabular}

* [4-787] JCPDS

** [25-1495] JCPDS with $a_{0}=4.12 \AA$

$* * *[25-1133]$ JCPDS

\section{Results and Discussion}

\subsection{Top VIEW}

3.1.1 Implantation at $1 \times 10^{17} \mathrm{~N}^{+} / \mathrm{cm}^{2}$. - The selected area electron diffraction pattern (SAED) in Figure 1a shows spots corresponding to a [110] fcc structure either of pure Al, $\mathrm{AlN}_{x}$ solid solution or of AlN compound. As a matter of fact, it is difficult to distinguish these two cubic phases since their crystallographic parameters are very close together (Tab. I). Some other spots (not indexed) are observed on the same figure and belong to the same phase rotated of $46^{\circ}$ from the precedent cubic crystallite. This corresponds to the diffraction of a disoriented microcrystallite. On the other hand, we measured by EDS on different large areas (400 $\mathrm{nm}$ diameter), an averaged $\mathrm{N} / \mathrm{Al}$ ratio close to 0.19 on this sample. Moreover, the $\mathrm{N} / \mathrm{Al}$ ratio value measured on some [110] fcc grains (Fig. 1a) was found to be equal to 0.20 . This allows us to assume the formation of solid solution $\mathrm{AlN}_{x}$ grains with cubic structure.

Besides the spots, arc of circle diffraction of weak intensity were observed on the first ring corresponding to the interplane distance $d=0.266 \mathrm{~nm}$ which is characteristic of the AlN hcp phase (Tab. II).

Thus it seems that small hexagonal AlN grains coexist with cubic grains of larger size. This result was confirmed by HRTEM observations combined with microbeam diffraction (MBD) which 


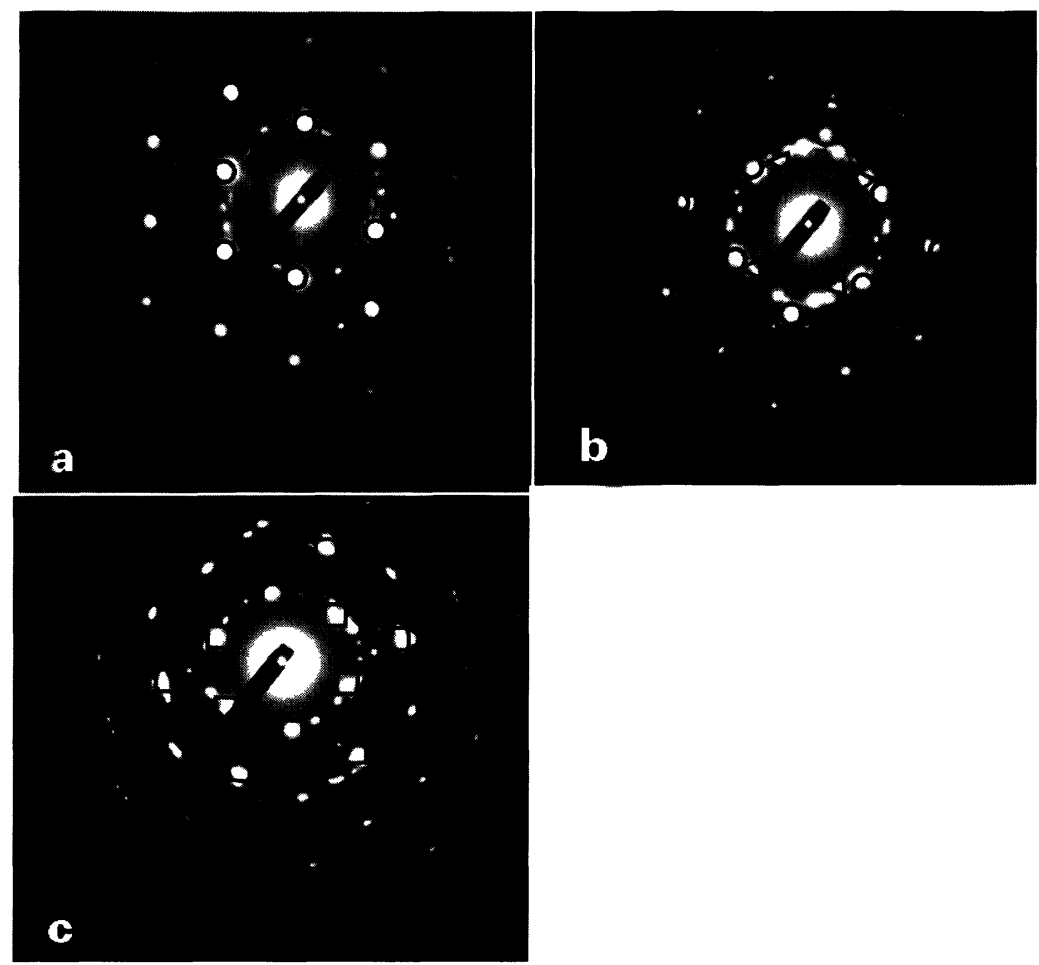

Fig. 1. - SAED patterns of implanted grids, with $1 \times 10^{17} \mathrm{~N}^{+} / \mathrm{cm}^{2}(\mathrm{a}), 2 \times 10^{17} \mathrm{~N}^{+} / \mathrm{cm}^{2}$ (b) and $3 \times$

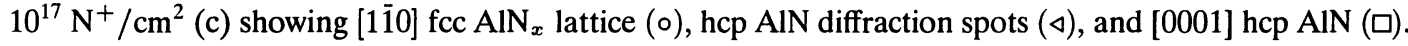

allows to reduce the beam size below the grain size. Thus, large grains of fcc structure with [011] and [213] preferred orientation with ill-defined morphology and variable contrast were observed besides small hexagonal AlN grains.

3.1.2 Implantation at $2 \times 10^{17} \mathrm{~N}^{+} / \mathrm{cm}^{2}$. - At medium dose $2 \times 10^{17} \mathrm{~N}^{+} / \mathrm{cm}^{2}$, the corresponding SAED pattern (Fig. 1b) shows the same [110] orientation for the cubic grains while diffraction spots attributed to the hcp AlN lattice become more important. Some grains ( $\varnothing \cong 100 \mathrm{~nm}$ ) of ill-defined morphology observed above belong now to the hcp AlN system while some others preserved their previous fcc structure. In Figure 2, one can see on such a grain atomic planes (A) separated by $0.230 \mathrm{~nm}$ which could be ascribed, with help of MBD, to [1102] hcp AlN. As for the previous implantation dose, small crystallites (4 to $8 \mathrm{~nm}$ ) with planes separated by $d=$ $0.264 \mathrm{~nm}$ (Fig. 2B) corresponding also to the hcp structure, are precipitated in the vicinity of these grains. Furthermore, some few multifaceted grains of hcp AlN, [0001] oriented, with a medium size ranging from 15 to $30 \mathrm{~nm}$, appear. The average $\mathrm{N} / \mathrm{Al}$ ratio measured on different large areas (400 $\mathrm{nm}$ diameter) of this grid was about 0.35 .

3.1.3 Implantation at $3 \times 10^{17} \mathrm{~N}^{+} / \mathrm{cm}^{2}$. - Finally, after implantation at $3 \times 10^{17} \mathrm{~N}^{+} / \mathrm{cm}^{2}$, one observes mainly precipitation of faceted hcp AlN grains with [0001] (Fig. 3a) and [1100] (Fig. 3b) preferred orientations. The ill-defined morphology subsists but is only associated with hcp AlN strongly oriented [0001] grains (Fig. 4). The preponderance of the hcp phase at this dose, could 

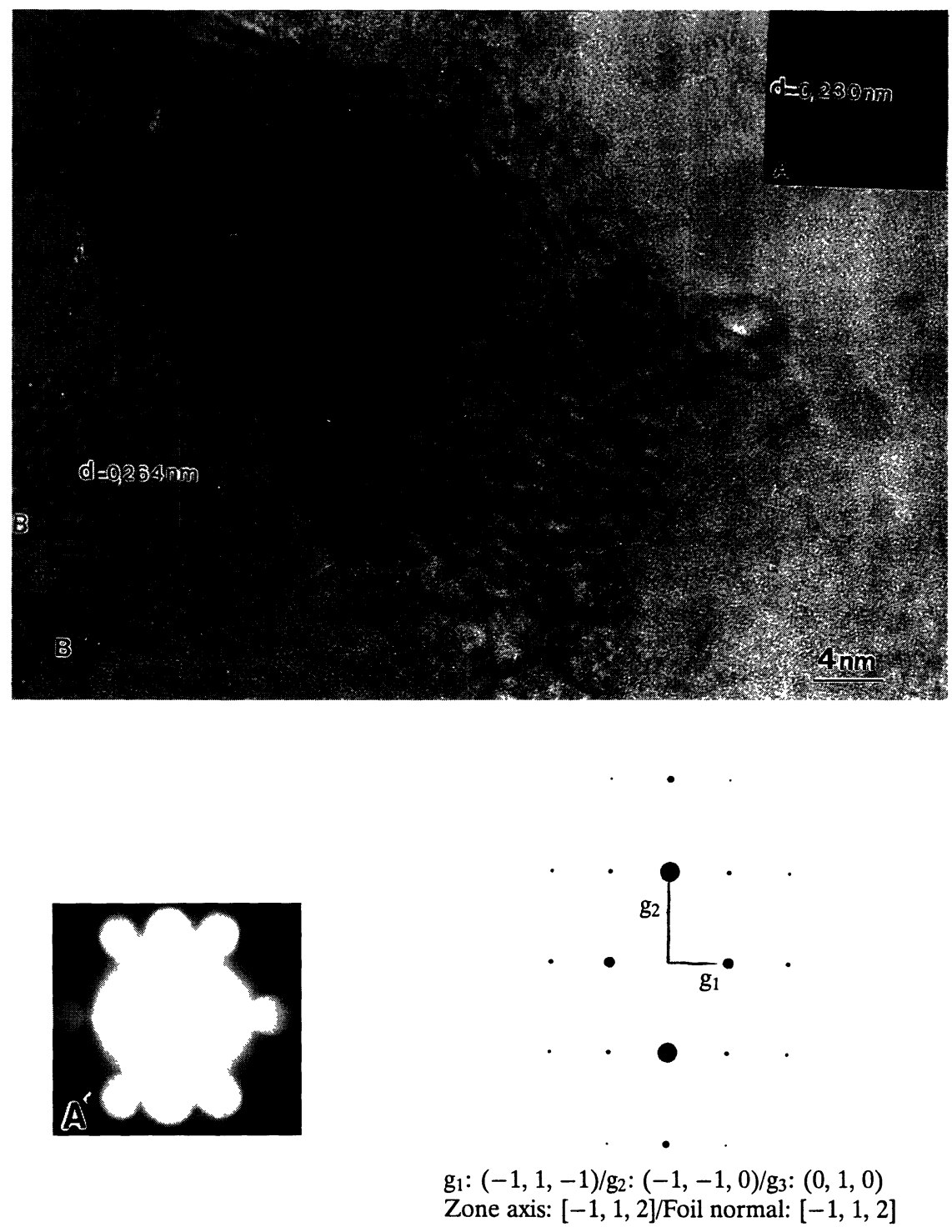

Fig. 2. - HREM image of an ill-defined hcp AlN grain observed on the $2 \times 10^{17} \mathrm{~N}^{+} / \mathrm{cm}^{2}$ implanted grid with its $\left(\mathrm{A}^{\prime}\right)$ MBED $(8.8 \mathrm{~nm})$ pattern whose indicate [1102] preferred orientation in agreement with its simulated pattern. (A) Image inset on the grain, (B) Image inset on $0.264 \mathrm{~nm}$ separated plane in the vicinity of the grain.

be also confirmed by SAED as shown in Figure 1c, which presents a spot pattern corresponding to hcp [0001] AlN.

EDXS microanalysis performed on grains of both cubic (Fig. 1b) and hexagonal (Fig. 1c) structure showed that the $\mathrm{N} / \mathrm{Al}$ ratio was about 0.3 on both grain types, while the mean value measured on wide areas $(400 \mathrm{~nm})$ of this grid was about 0.53 . 

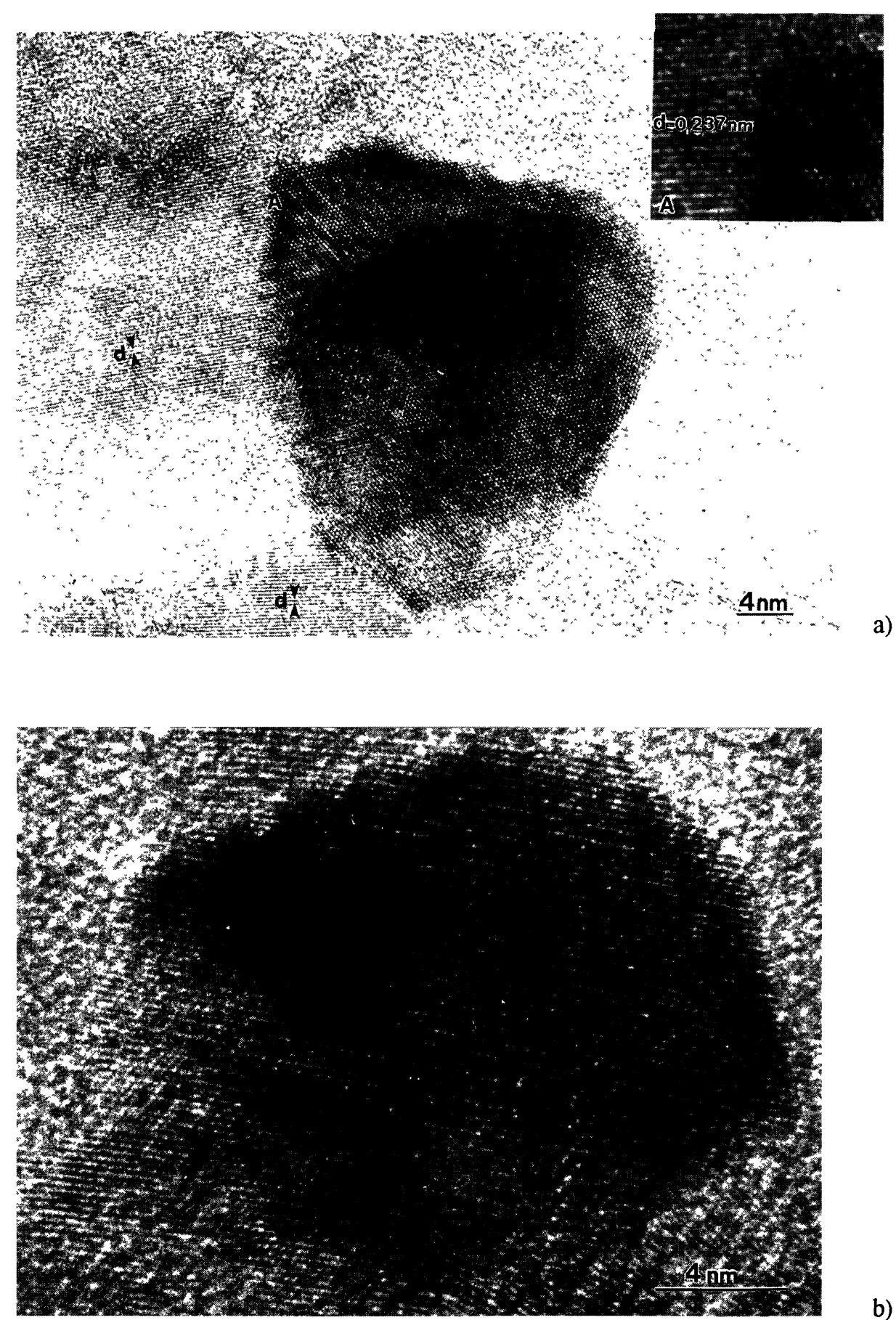

b)

Fig. 3. - HREM image of two hcp AIN faceted grains observed on the $3 \times 10^{17} \mathrm{~N}^{+} / \mathrm{cm}^{2}$ implanted grid showing two atomic lattices with [0001] (a) and [1100] (b) preferred orientations. The corresponding diffraction patterns and their simulation are given in Figure $3 \mathrm{a}$ bis and Figure $3 \mathrm{~b}$ bis. 

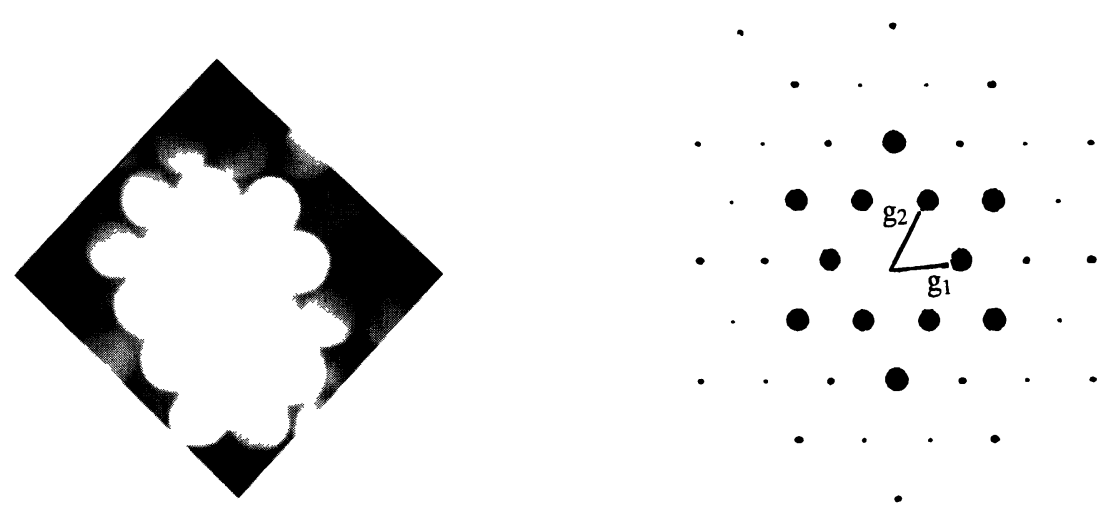

$\mathrm{g}_{1}:(1,0,0) / \mathrm{g}_{2}:(0,1,0) / \mathrm{g}_{3}:(0,0,1)$

Zone axis: $[0,0,1] /$ Foil normal: $[0,0,1]$

(a bis)
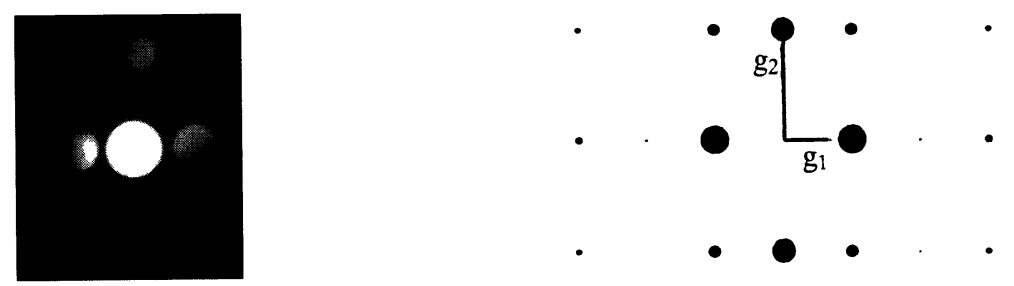

$\mathrm{g}_{1}:(0,0,1) / \mathrm{g}_{2}:(-1,-1,0) / \mathrm{g}_{3}:(0,-1,0)$

Zone axis: $[1,-1,0] /$ Foil normal: $[1,-1,0]$

(b bis)

Fig. 3. - continued

Table III. - Concentration ratios N/Al measured by EDXS microanalysis on the Al implanted films.

\begin{tabular}{|c|c|c|}
\hline $\begin{array}{c}\text { Doses } \times 10^{17} \\
\left(\mathrm{~N}^{+} / \mathrm{cm}^{2}\right)\end{array}$ & $\mathrm{N} / \mathrm{Al}^{\mathrm{a}}$ & $\mathrm{N} / \mathrm{Al}^{\mathrm{b}}$ \\
\hline 1 & 0.19 & 0.28 \\
\hline 2 & 0.35 & - \\
\hline 3 & 0.53 & 0.77 \\
\hline
\end{tabular}

${ }^{\text {a }}$ top view analysis $(0.4 \mu \mathrm{m})$.

${ }^{b}$ cross section analysis. 


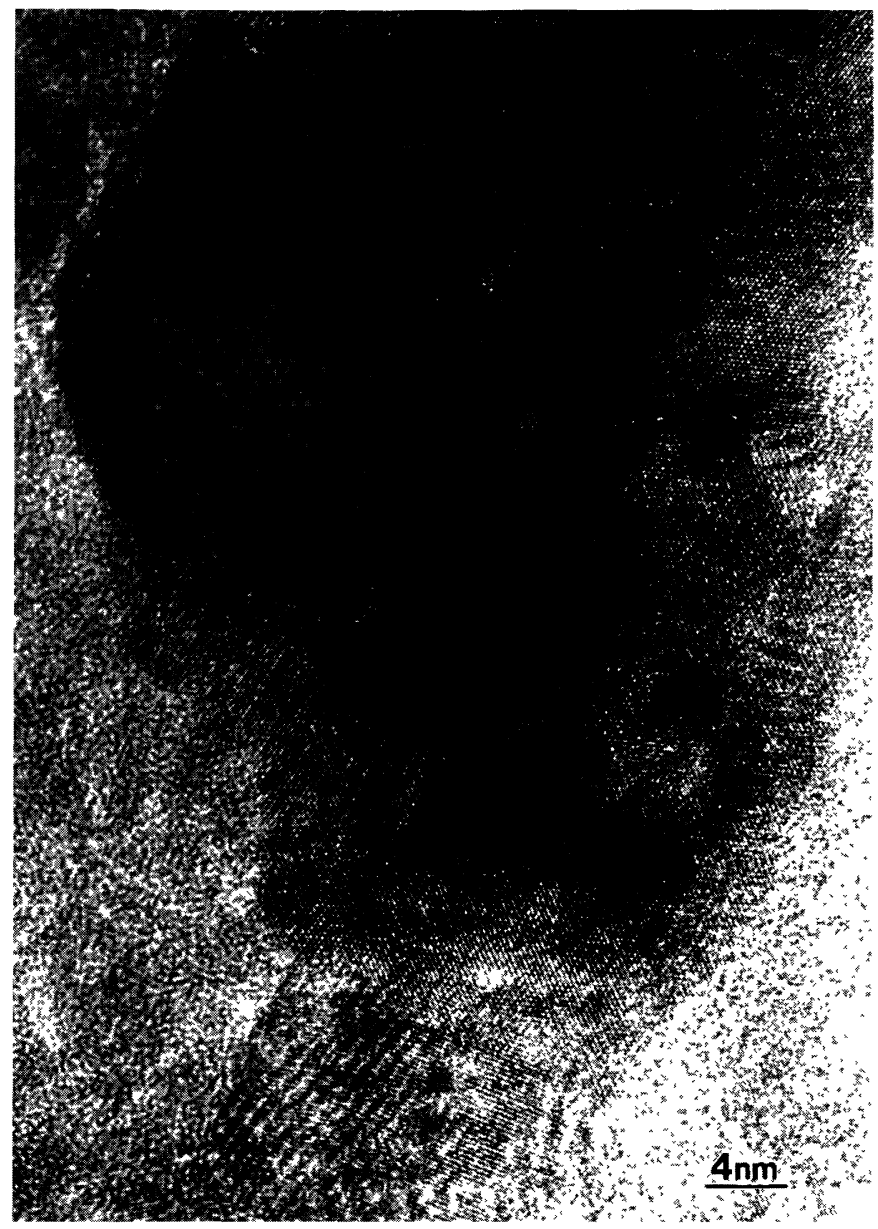

Fig. 4. - HREM image of a [0001] hcp AlN large grain with ill-defined morphology observed at $3 \times 10^{17} \mathrm{~N}^{+} / \mathrm{cm}^{2}$ dose.

3.2 Cross SeCtion View. - The high dose $\left(3 \times 10^{17} \mathrm{~N}^{+} / \mathrm{cm}^{2}\right)$ implanted film presents a layered structure (region 1, 2 and 3) consisting of (1) an amorphous aluminum oxide overlayer, (2) a noncontinuous layer containing AlN crystallites and (3) a carbon layer (Fig. 5). The intermediate layer (2) consists of three sublayers characterized by their nitrogen content. The upper (A) and lower (C) sublayers coincide with the flanks of the $\mathrm{N}$ concentration profile established by EDXS microanalysis. The maximum nitrogen concentration for the low and high dose is localized at $47 \mathrm{~nm}$ and the corresponding maximum concentration ratios $\mathrm{N} / \mathrm{Al}$ are respectively 0.28 and 0.77 . These values are in rather good agreement with the calculated ones. It should be noticed that these values are greater than those measured on top views for the corresponding doses, because on cross section we measured the true N/Al implantation maximum, while we measured on top views a mean $\mathrm{N} / \mathrm{Al}$ value provided from the whole depth of the $\mathrm{Al}$ layer.

The microbeam diffraction realized with a spot size of $4.4 \mathrm{~nm}$ on these regions shows that only the intermediate layer diffracts and evidences mainly the presence of hcp AlN grains in the high 


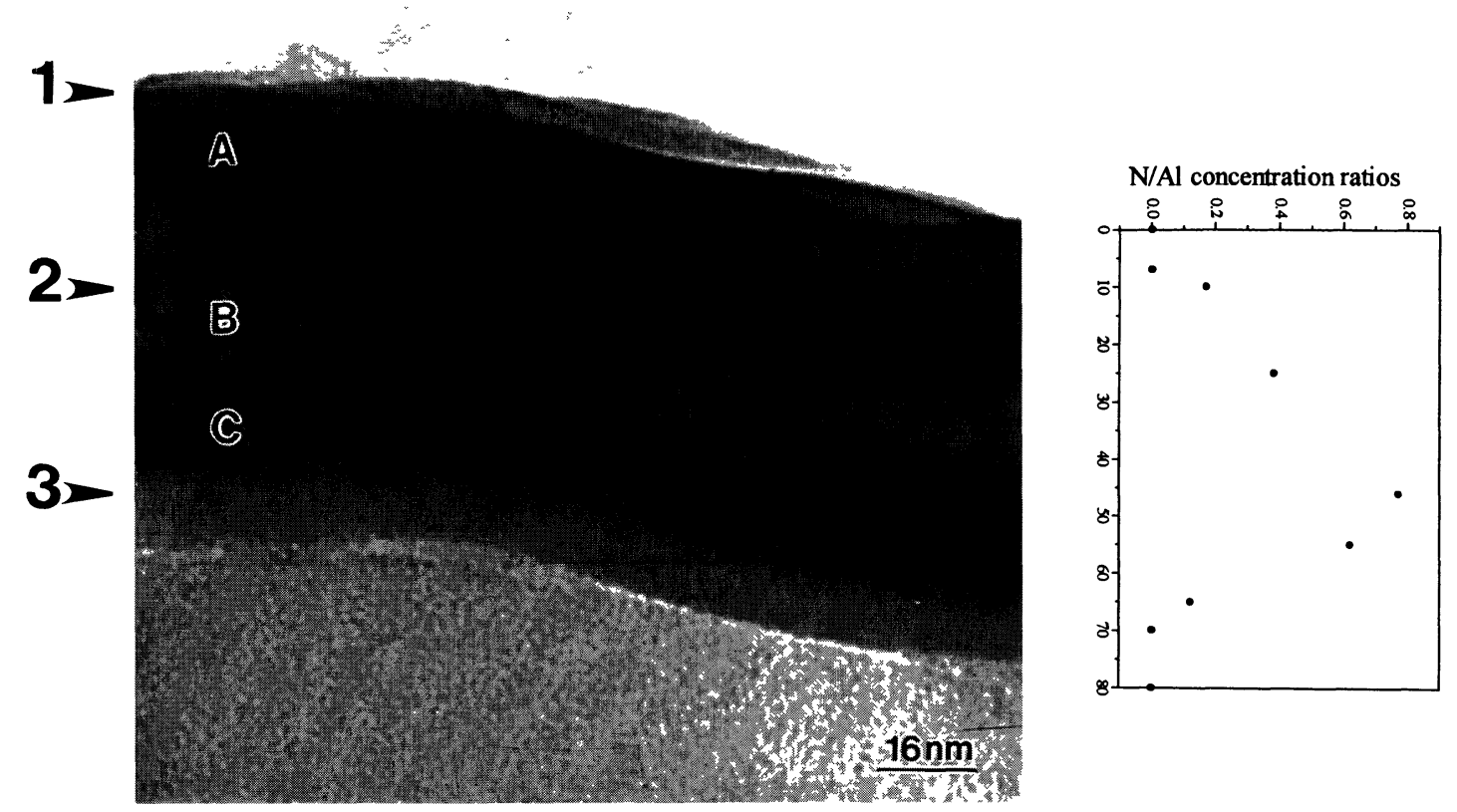

Fig. 5. - Cross section view micrograph of the $3 \times 10^{17} \mathrm{~N}^{+} / \mathrm{cm}^{2}$ implanted grid correlated with nitrogen concentration profile established by EDXS.

dose case. In the same way mixed spots belonging to cubic $\mathrm{Al}$ and hexagonal $\mathrm{AlN}$ are observed on the nanodiffraction pattern in the low dose case.

\section{Conclusion}

This HRTEM study allows us to differentiate two morphologic types and, with the help of EDXS microanalysis, to measure the N/Al concentration ratios on different regions of the grids.

The first morphology was present in the three implantation cases. It was manifested by an illdefined aspect corresponding, in the case of $1 \times 10^{17} \mathrm{~N}^{+} / \mathrm{cm}^{2}$ implanted grid, to fcc grains with [110] as the main orientation. When increasing the nitrogen dose, this morphology corresponds mainly to hcp AlN [0001].

The second morphology appears at medium dose and was enhanced at $3 \times 10^{17} \mathrm{~N}^{+} / \mathrm{cm}^{2}$. It can be distinguished by its faceted edges and corresponds to hcp AlN [0001] mainly. The origin of these faceted grains seems to be related to the growth of some hcp AlN nanometric nuclei precipitated in the vicinity of large ill-defined grains.

By means of EDXS microanalysis, we measured N/Al ratios, for each implanted grid on top views as well as on cross sections. Since we found, by top view analysis, 0.20 and 0.30 as N/Al ratios on fcc grains for low dose, we assume the formation of grains belonging to a $\mathrm{AlN}_{x}$ solid solution. Besides, we measured, by cross section analysis, N/Al concentration ratios going through a maximum localized at $47 \mathrm{~nm}$. Finally, aluminum nitride grains of cubic or hexagonal close packed structure were detected with substoichiometric composition. 


\section{References}

[1] Morita M., Isogai S., Tsubouchi K. and Mikoshiba N., Appl. Phys. Lett. 38 (1981) 50.

[2] Kawabata A., Jpn J. Appl. Phys. 23, Suppl. 1, Part 1 (1984) 17.

[3] Murayama Y. and Kashiwagi K., J. Vac. Sci. Technol. 17 (1980) 796.

[4] Pavlov P.V., Zorin E.J.,Tetelbamn P.I., Lesnikov V.P., Ryzhkov G.M. and Pavlov A.V., Phys. Status Solidi A 19 (1973) 373.

[5] Belii I.M., Komarov F.F., Tishkov V.S. and Yankovskii V.M., Phys. Status Solidi A 45 (1978) 343.

[6] Rauschenbach B., Kolitsch A. and Richter E., Thin Solid Films 109 (1983) 37.

[7] Ohira S. and Iwaki M., Mater. Sci. Eng. 90 (1987) 143.

[8] Bykov V.N., Troyan V.A., Zobrovtseva G.G. and Khaimovich V.S., Phys. Status Solidi A 32 (1975) 53.

[9] Matthews A.P., Iwaki M., Horino Y., Satou M. and Yabe K., Nucl. Inst. Meth. B 59/60 (1991) 671.

[10] Seghrouchni Z., Mosser A., Grob J.J., Broll N. and Cornet A., J. Phys. France, in press.

[11] Ziegler J.F., Biersack J.P. and Littmark U., in: The Stopping and Ranges of Ions in Solids, vol.1, J.F. Ziegler Ed. (Pergman, New York, 1985).

[12] Stadelmann P., Ultramicroscopy 21 (1987) 131. 